\title{
Application of the DTM to Nonlinear Cases Arising in Fluid Flows with Variable Viscosity
}

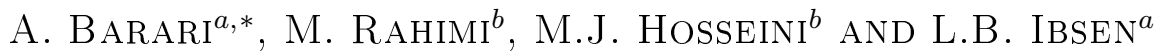 \\ ${ }^{a}$ Department of Civil Engineering, Aalborg University, Sohngårdsholmsvej 57, Aalborg 9000, Aalborg, Denmark \\ ${ }^{b}$ Department of Mechanical Engineering, Babol University of Technology, P.O. Box 484, Babol, Iran
}

(Received May 19, 2011; in final form May 14, 2012)

\begin{abstract}
This paper employs the differential transformation method to investigate two nonlinear ordinary differential systems for plane coquette flow having variable viscosity and thermal conductivity. The concept of differential transformation is briefly introduced, and then differential transformation method is employed to derive solutions of nonlinear equation systems. The results of differential transformation method are compared with those ones obtained by Adomian decomposition method to verify the accuracy of proposed method. The results reveal that the differential transformation method can achieve suitable results in predicting the solution of such problems.
\end{abstract}

PACS: 47.35.Pq, 42.65.Wi

\section{Introduction}

In recent years some researchers used new methods to solve ordinary and partial differential equation with or without initial and boundary conditions [1-5]. Integral transformation methods such as the Laplace or Fourier transformation methods are widely used in engineering problems. These methods transform differential equations into algebraic equations which are easier to deal with. However, integral transformation methods are more complex and difficult when applying to nonlinear problems. The differential transformation method (DTM) was first applied in the engineering domain by Zhou [6]. This method is based on the Taylor expansion. It constructs an analytical solution in the form of a polynomial. It is different from the traditional high order Taylor series method, which requires symbolic computation of the necessary derivatives of the data functions. The Taylor series method is computationally taken long time for large orders. The mentioned method is an iterative procedure for obtaining analytic Taylor series solutions of differential equations. DTM has the inherent ability to deal with nonlinear problems, and consequently Chiou [7] applied the Taylor transformation to solve nonlinear vibration problems. Furthermore, the method described above, may be employed for the solution of both ordinary and partial differential equations. Jang et al. [8] applied the two-dimensional DTM to solve the partial differential equations. Finally, Hassan [9] adopted the DTM to solve case study problems. This method was successfully applied to various application problems [10-13]. Recently approximate analytical methods are widely used in different fields which have appeared in literature [14-24].

* corresponding author; e-mail: ab@civil.aau.dk, amin78404@yahoo.com
In the present study, we have used DTM for nonlinear ordinary differential systems for plane coquette flow, which are derived from the Navier-Stokes equations, considering the following assumptions:

a) steady flow,

b) one dimension,

c) negligible body force,

d) negligible pressure gradient.

The current results are then compared with those derived from the variational iteration method (VIM), homotopy perturbation method (HPM) and perturbation method [25] in order to verify the accuracy of the proposed method.

\section{Differential transformation method}

DTM is a straight forward method in the form of a polynomial for solving differential equations initially introduced by Zhou [6], reducing the size of computational volume compared to the Taylor series method. Suppose that $u(t)$ is an analytic function in a domain $D$ and $t=t_{i}$ represent any point in $D$. The function $u(t)$ is then represented by one power series whose center is located at $t_{i}$. The Taylor series expansion function of $u(t)$ is of the form [10-12]:

$$
u(t)=\sum_{k=0}^{\infty} \frac{\left(t-t_{i}\right)^{k}}{k !}\left[\frac{\mathrm{d}^{k} u(t)}{\mathrm{d} t^{k}}\right]_{t=t_{i}} \forall t \in D .
$$

The particular case of Eq. (1) when $t_{i}=0$ is referred to the Maclaurin series of $u(t)$ is expressed as

$$
u(t)=\sum_{k=0}^{\infty} \frac{t^{k}}{k !}\left[\frac{\mathrm{d}^{k} u(t)}{\mathrm{d} t^{k}}\right]_{t=0} \forall t \in D .
$$

As explained in [10-12] the differential transformation of the function $u(t)$ is defined as follows: 


$$
U(k)=\sum_{k=0}^{\infty} \frac{H^{k}}{k !}\left[\frac{\mathrm{d}^{k} u(t)}{\mathrm{d} t^{k}}\right]_{t=0} \forall t \in D,
$$

where $u(t)$ is the original function and $U(k)$ is the transformed function. The differential spectrum of $U(k)$ is confined within the interval $t \in[0, H]$, in which $H$ is a constant. The differential inverse transform of $U(k)$ is defined as follows:

$$
u(t)=\sum_{k=0}^{\infty}\left(\frac{t}{H}\right)^{k} U(K) .
$$

It is clear that the concept of differential transformation is based upon the Taylor series expansion. The values of function $U(k)$ at values of argument $k$ are referred to as discrete, i.e. $U(0)$ is known as the zero discrete, $U(1)$ as the first discrete, etc. The function $u(t)$ consists of the $T$-function $U(k)$, and its value given by the sum of the $T$-function with $(t / H)^{k}$ as its coefficient. In real applications, at the right choice of constant $H$ and the larger values of argument $k$, the discrete of spectrum reduces rapidly.

The function $u(t)$ is expressed by a finite series and Eq. (4) can be written as

$$
u(t)=\sum_{k=0}^{n}\left(\frac{t}{H}\right)^{k} U(K) .
$$

Mathematical operations performed by differential transformation method are listed in Table I.

Operations of the one-dimensional

TABLE I differential transformation.

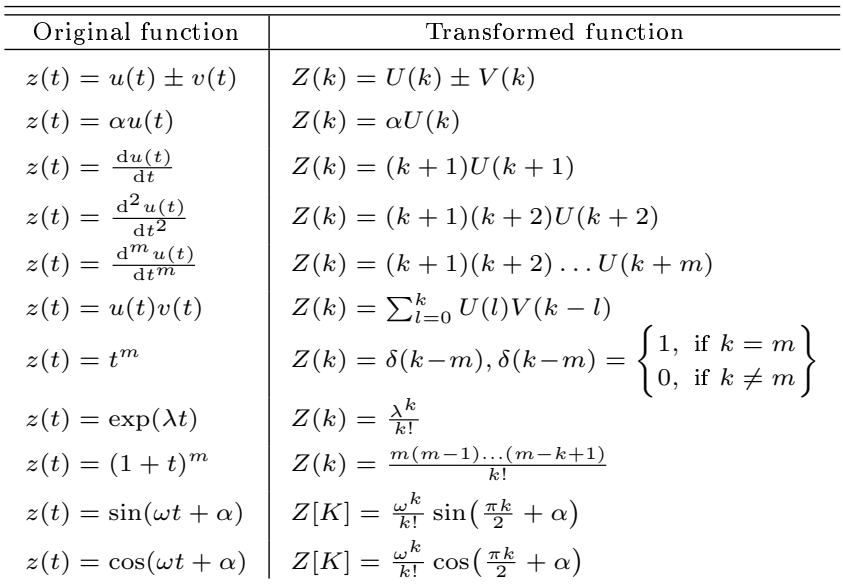

\section{Plane coquette flow with variable viscosity (first problem)}

\subsection{Description of the first problem}

The characteristic behavior of fluids is extremely dependent on the viscosity. The fluids are set to be Newtonianfluids if the viscosity of thefluids remains constant and is independent of the applied shear stress. In the case of the non-Newtonian fluids, when the shear rate is varied, the shear stress does not vary proportionally.

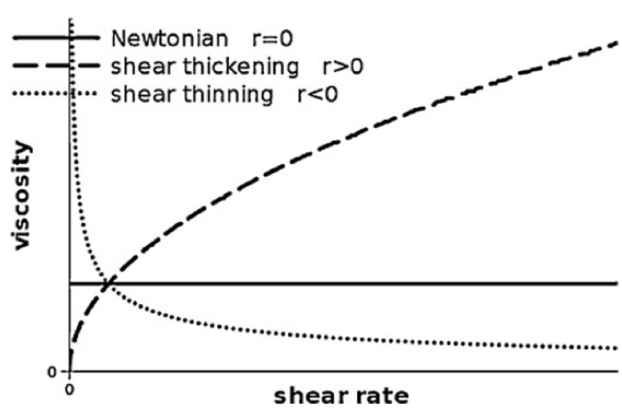

Fig. 1. Various types of fluids based on stress and on viscosity [26].

Figure 1 shows that how the shear rate affects the shear stress and viscosity.

Consider the steady flow of an incompressible Newtonian fluid between two infinite parallel plates separated by a specific distance.

Each plate is maintained at temperature $T_{0}$. The lower plate is stationary while the upper plate moves with a uniform velocity $V$. The thermal conductivity of the fluid is assumed to be constant, but the viscosity is allowed to vary. The pertinent momentum and energy equations are [27]:

$$
\begin{aligned}
& \left\{\begin{array}{l}
\frac{\mathrm{d}}{\mathrm{d} y}\left(\mu \frac{\mathrm{d} u}{\mathrm{~d} y}\right)=0, \\
\frac{\mathrm{d}^{2} T}{\mathrm{~d} y^{2}}+\frac{\mu}{k}\left(\frac{\mathrm{d} u}{\mathrm{~d} y}\right)^{2}=0,
\end{array}\right. \\
& y=0 \rightarrow u=0, \quad T=T_{0} \text {, } \\
& y=a \rightarrow u=V, \quad T=T_{0},
\end{aligned}
$$

where $u, \mu$ and $k$ are axial velocity, viscosity and thermal conductivity, respectively.

Let the viscosity vary exponentially with temperature as

$$
\mu=\mu_{0} \mathrm{e}^{-\alpha\left(T-T_{0}\right)},
$$

where $\mu_{0}$ is the viscosity and $\alpha$ is constant.

To solve this equation, we use the following non-dimensional parameters:

$$
\begin{aligned}
& \theta=\frac{T-T_{0}}{T_{0}}, \quad Y=\frac{y}{a}, \quad U=\frac{u}{V}, \quad \beta=\alpha T_{0}, \\
& B r=\frac{\mu_{0} V^{2}}{k T_{0}},
\end{aligned}
$$

where $\theta$ is dimensionless temperature, $Y$ is dimensionless height, $U$ is dimensionless velocity, $\beta$ is constant, $B r$ is the Brinkman number.

By substitution the foregoing parameters into Eq. (6), it gives us 


$$
\begin{aligned}
& \left\{\begin{array}{l}
\frac{\mathrm{d}}{\mathrm{d} Y}\left(\mathrm{e}^{-\beta \theta} \frac{\mathrm{d} U}{\mathrm{~d} Y}\right)=0, \\
\frac{\mathrm{d}^{2} \theta}{\mathrm{d} Y^{2}}+B r \mathrm{e}^{-\beta \theta}\left(\frac{\mathrm{d} U}{\mathrm{~d} Y}\right)^{2}=0 .
\end{array}\right. \\
& Y=0 \rightarrow U=0, \quad \theta=0 \text {, } \\
& Y=1 \rightarrow U=1, \quad \theta=0 .
\end{aligned}
$$

We use the following expansion for $\mathrm{e}^{-\beta \theta}$ :

$$
\mathrm{e}^{-\beta \theta}=1-\beta \theta+\ldots
$$

\subsection{Solution of the first problem with differential transformation method}

Now, applying DTM into Eq. (11) and taking the differential transform of Eq. (11) with respect to $y$, and considering $H=1$, gives

$$
\begin{aligned}
& (k+1)(k+2) U(k+2) \\
& -\beta \sum_{i=0}^{k}(i+1) \theta(i+1)(k-i+1) U(k-i+1) \\
& -\beta \sum_{i=0}^{k} \theta(i)(k-i+1)(k-i+2) U(k-i+2)=0, \\
& (k+1)(k+2) \theta(k+2) \\
& +B r \sum_{i=0}^{k}(i+1) U(i+1)(k-i+1) U(k-i+1) \\
& -\beta B r \sum_{i=0}^{k} \theta(k-i) \sum_{p=0}^{i}(p+1) U(p+1)(i-p+1) \\
& \times U(i-p+1)=0 .
\end{aligned}
$$

By applying transformation on boundary condition (12), the following is obtained:

$$
U(0)=0, \quad \theta(0)=0
$$

and the other boundary conditions will be as follows:

$$
U(1)=a, \quad \theta(1)=b,
$$

where $a$ and $b$ are constants, which will be calculated by considering another boundary condition in (13) in point $y=1$.

So the following equations will be obtained:

$$
\begin{aligned}
& \left\{\begin{array}{l}
\theta(2)=-\frac{1}{2} B r a^{2}, \\
U(2)=\frac{1}{2} \beta b a,
\end{array}\right. \\
& \left\{\begin{array}{l}
\theta(3)=-\frac{1}{6} \beta B r b a^{2}, \\
U(3)=\frac{1}{3} \beta^{2} b^{2} a-\frac{1}{6} \beta B r a^{3},
\end{array}\right. \\
& \left\{\begin{array}{l}
\theta(4)=-\frac{1}{12} \beta^{2} B r b^{2} a^{2}-\frac{1}{2} B r a\left(\frac{1}{3} \beta^{2} b^{2} a-\frac{1}{6} \beta B r a^{3}\right) \\
-\frac{1}{24} \beta B r^{2} a^{4}, \\
U(4)=\frac{3}{4} \beta b\left(\frac{1}{3} \beta^{2} b^{2} a-\frac{1}{6} \beta B r a^{3}\right)-\frac{1}{6} \beta^{2} B r a^{3} b,
\end{array}\right.
\end{aligned}
$$

$$
\left\{\begin{array}{l}
\theta(5)=-\frac{7}{120} \beta^{2} B r^{2} b a^{4}-\frac{2}{5} B r a\left[\frac { 3 } { 4 } \beta b \left(\frac{1}{3} \beta^{2} b^{2} a\right.\right. \\
\left.\left.-\frac{1}{6} \beta B r a^{3}\right)-\frac{1}{6} \beta^{2} B r b a^{3}\right]+\frac{1}{20} \beta^{3} B r b^{3} a^{2} \\
U(5)=\frac{1}{5} \beta a\left[\frac{1}{12} \beta^{2} B r b^{2} a^{2}-\frac{1}{2} B r a\left(\frac{1}{3} \beta^{2} b^{2} a\right.\right. \\
\left.\left.-\frac{1}{6} \beta B r a^{3}\right)-\frac{1}{24} \beta B r^{2} a^{4}\right]+\frac{4}{5} \beta b\left[\frac { 3 } { 4 } \beta b \left(\frac{1}{3} \beta^{2} b^{2} a\right.\right. \\
\left.\left.-\frac{1}{6} \beta B r a^{3}\right)-\frac{1}{6} \beta^{2} B r a^{3} b\right]-\frac{3}{10} \beta B r a^{2}\left(\frac{1}{3} \beta^{2} b^{2} a\right. \\
\left.-\frac{1}{6} \beta B r a^{3}\right)-\frac{1}{30} \beta^{3} B r b^{2} a^{3} .
\end{array}\right.
$$

The foregoing process is continuous. By substituting Eq. (18) into the main equation based on DTM, the following solution would be obtained:

$$
\begin{aligned}
& \theta(y)=b y-\frac{1}{2} B r a^{2} y^{2}-\frac{1}{6} \beta B r b a^{2} y^{3}+\left[\frac{1}{12} \beta^{2} B r a^{2} b^{2}\right. \\
& \left.-\frac{1}{2} B r a\left(\frac{1}{3} \beta^{2} a b^{2}-\frac{1}{6} \beta B r a^{3}\right)-\frac{1}{24} \beta B r^{2} a^{4}\right] y^{4} \\
& +\left\{-\frac{7}{120} \beta^{2} B r^{2} a^{4} b-\frac{2}{5} B r a\left[\frac { 3 } { 4 } \beta b \left(\frac{1}{3} \beta^{2} b^{2} a\right.\right.\right. \\
& \left.\left.\left.-\frac{1}{6} \beta B r a^{3}\right)-\frac{1}{6} \beta^{2} B r a^{3} b\right]+\frac{1}{20} \beta^{3} B r b^{3} a^{2}\right\} y^{5} \\
& +\ldots
\end{aligned}
$$

and

$$
\begin{aligned}
& U(y)=a y+\frac{1}{2} \beta b a y^{2}+\left(\frac{1}{3} \beta^{2} a b^{2}-\frac{1}{6} \beta B r a^{3}\right) y^{3} \\
& +\left[\frac{3}{4} \beta b\left(\frac{1}{3} \beta^{2} b^{2} a-\frac{1}{6} \beta B r a^{3}\right)-\frac{1}{6} \beta^{2} B r a^{3} b\right] y^{4} \\
& +\left\{\frac { 1 } { 5 } \beta \left[\frac{1}{12} \beta^{2} B r b^{2} a^{2}-\frac{1}{2} B r a\left(\frac{1}{3} \beta^{2} b^{2} a-\frac{1}{6} \beta B r a^{3}\right)\right.\right. \\
& \left.-\frac{1}{24} \beta B r^{2} a^{4}\right] a+\frac{4}{5} \beta b\left[\frac{3}{4} \beta b\left(\frac{1}{3} \beta^{2} b^{2} a-\frac{1}{6} \beta B r a^{3}\right)\right. \\
& \left.-\frac{1}{6} \beta^{2} B r a^{3} b\right]-\frac{3}{10} \beta B r a^{2}\left(\frac{1}{3} \beta^{2} b^{2} a-\frac{1}{6} \beta B r a^{3}\right) \\
& \left.-\frac{1}{30} \beta^{3} B r a^{3} b^{2}\right\} y^{5}+\ldots
\end{aligned}
$$

Substitution of boundary condition (13) into Eq. (19) gives us $a$ and $b$ through the following equations:

$$
\begin{aligned}
& \theta(1)=b-\frac{1}{2} B r a^{2}-\frac{1}{6} \beta B r b a^{2}-\frac{1}{12} \beta^{2} B r a^{2} b^{2} \\
& +\frac{1}{24} \beta B r^{2} a^{4}+\frac{7}{120} \beta^{2} B r^{2} a^{4} b-\frac{1}{20} \beta^{3} B r a^{2} b^{3} \\
& +\ldots=0
\end{aligned}
$$

and 


$$
\begin{aligned}
& U(1)=a+\frac{1}{2} \beta b a+\frac{1}{3} \beta^{2} b^{2} a-\frac{1}{6} \beta B r a^{3}+\frac{1}{4} \beta^{3} b^{3} a \\
& -\frac{7}{24} \beta^{2} B r a^{3} b-\frac{23}{60} \beta^{3} B r a^{3} b^{2}+\frac{7}{120} \beta^{2} B r^{2} a^{5} \\
& +\frac{1}{5} \beta^{4} a b^{4}+\ldots=1 .
\end{aligned}
$$

Solving Eqs. (21) and (22), gives us the value of $a$ and $b$. These values are too long to be shown in this paper. By substituting $a$ and $b$ obtained above, into Eq. (19) and Eq. (20), we can find the expressions of $\theta(y)$ and $U(y)$.

\section{The second case}

\subsection{Description of second problem}

As a more realistic analysis of plane coquette flow, we should consider that viscosity and thermal conductivity can vary with a temperature. In this case the governing equations are [27]:

$$
\left\{\begin{array}{l}
\frac{\mathrm{d}}{\mathrm{d} Y}\left(\frac{k}{k_{0}} \frac{\mathrm{d} \theta}{\mathrm{d} Y}\right)+\varepsilon \frac{\mu}{\mu_{0}}\left(\frac{\mathrm{d} U}{\mathrm{~d} Y}\right)^{2}=0 \\
\frac{\mathrm{d}}{\mathrm{d} Y}\left(\frac{\mu}{\mu_{0}} \frac{\mathrm{d} U}{\mathrm{~d} Y}\right)=0
\end{array}\right.
$$

where

$$
\frac{k}{k_{0}}=1+\alpha_{1} \theta+\alpha_{2} \theta^{2}
$$

and

$$
\frac{\mu_{0}}{\mu}=1+\beta_{1} \theta+\beta_{2} \theta^{2} .
$$

We use the following expansion for $\mu / \mu_{0}$ :

$$
\begin{aligned}
& \frac{\mu}{\mu_{0}}=\frac{1}{1+\beta_{1} \theta+\beta_{2} \theta^{2}}=1-\beta_{1} \theta+\ldots \\
& Y=0 \rightarrow U=0, \quad \theta=0, \\
& Y=1 \rightarrow U=1, \quad \theta=0 .
\end{aligned}
$$

\subsection{Solution of second problem by applying DTM}

Now, applying DTM into Eq. (23) and taking the differential transform of Eq. (23) with respect to $y$, and considering $H=1$ gives

$$
\begin{aligned}
& (k+1)(k+2) U(k+2) \\
& -\beta_{1} \sum_{i=0}^{k}(i+1) \theta(i+1)(k-i+1) U(k-i+1) \\
& -\beta_{1} \sum_{i=0}^{k} \theta(i)(k-i+1)(k-i+2) U(k-i+2)=0,
\end{aligned}
$$

$$
(k+1)(k+2) \theta(k+2)
$$$$
+\alpha_{1} \sum_{i=0}^{k}(i+1) \theta(i+1)(k-i+1) \theta(k-i+1)
$$$$
+2 \alpha_{2} \sum_{i=0}^{k} \theta(k-i)
$$

$$
\begin{aligned}
& \times \sum_{p=0}^{i}(p+1) \theta(p+1)(i-p+1) \theta(i-p+1) \\
& +\alpha_{2} \sum_{i=0}^{k}(k-i+1)(k-i+2) \theta(k-i+2) \\
& \times \sum_{p=0}^{i} \theta(p) \theta(i-p) \\
& +\varepsilon \sum_{i=0}^{k}(k-i+1) \theta(k-i+1)(i+1) \theta(i+1) \\
& -\varepsilon \beta_{1} \sum_{i=0}^{k}(k-i) \\
& \times \sum_{p=0}^{i}(p+1) \theta(p+1)(i-p+1) U(i-p+1)=0 .
\end{aligned}
$$

From boundary conditions $(27)$ in point $y=0$ we have

$$
U(0)=0, \quad \theta(0)=0 .
$$

Other boundary conditions are considered as follows:

$$
U(1)=a, \quad \theta(1)=b,
$$

where $a$ and $b$ are constants, and we will calculate them by considering another boundary condition in (8) at point $y=1$.

We will have

$$
\begin{aligned}
& \left\{\begin{array}{l}
\theta(2)=-\frac{1}{2} \alpha_{1} b^{2}-\frac{1}{2} \varepsilon a^{2} \\
U(2)=\frac{1}{2} \beta_{1} b a
\end{array}\right. \\
& \left\{\begin{array}{l}
\theta(3)=-\frac{1}{6} \beta_{1} \varepsilon b a^{2}-\alpha_{1} b\left(-\frac{1}{2} \alpha_{1} b^{2}-\frac{1}{2} \varepsilon a^{2}\right)-\frac{1}{3} \alpha_{2} b^{3} \\
U(3)=\frac{1}{3} \beta_{1}^{2} b^{2} a+\frac{1}{3} \beta_{1} a\left(-\frac{1}{2} \alpha_{1} b^{2}-\frac{1}{2} \varepsilon a^{2}\right)
\end{array}\right. \\
& \left\{\begin{array}{l}
\theta(4)=-\frac{1}{2} \varepsilon a\left[\frac{1}{3} \beta_{1}^{2} b^{2} a+\frac{1}{3} \beta_{1}\left(-\frac{1}{2} \alpha_{1} b^{2}-\frac{1}{2} \varepsilon a^{2}\right) a\right] \\
-\alpha_{1} b\left\{-\frac{1}{6} \varepsilon \beta_{1} b a^{2}-\alpha_{1} b\left(-\frac{1}{2} \alpha_{1} b^{2}-\frac{1}{2} \varepsilon a^{2}\right)\right. \\
\left.-\frac{1}{3} \alpha_{2} b^{3}\left[-\frac{1}{2} \alpha_{1}\left(-\frac{1}{2} \alpha_{1} b^{2}-\frac{1}{2} \varepsilon a^{2}\right)\right]\right\} \\
-\alpha_{2} b^{2}\left(-\frac{1}{2} \alpha_{1} b^{2}-\frac{1}{2} \varepsilon a^{2}\right) \\
+\frac{1}{12} \varepsilon \beta_{1}^{2} b^{2} a^{2}+\frac{1}{12} \varepsilon \beta_{1} a^{2}\left(-\frac{1}{2} \alpha_{1} b^{2}-\frac{1}{2} \varepsilon a^{2}\right) \\
U(4)=\frac{3}{4} \beta_{1} b\left[\frac{1}{3} \beta_{1}^{2} b^{2} a+\frac{1}{3} \beta_{1}\left(-\frac{1}{2} \alpha_{1} b^{2}-\frac{1}{2} \varepsilon a^{2}\right) a\right] \\
+\frac{1}{4} \beta_{1}^{2} b a\left(-\frac{1}{2} \alpha_{1} b^{2}-\frac{1}{2} \varepsilon a^{2}\right)+\frac{1}{4} \beta_{1}\left[-\frac{1}{6} \varepsilon \beta_{1} b a^{2}\right. \\
\left.-\alpha_{1} b\left(-\frac{1}{2} \alpha_{1} b^{2}-\frac{1}{2} \varepsilon a^{2}\right)-\frac{1}{3} \alpha_{2} b^{3}\right] a
\end{array}\right.
\end{aligned}
$$

The foregoing process is continuous. By substituting Eq. (23) into the main equation based on DTM, the following solution would be obtained:

$$
\theta(y)=b y+\left(-\frac{1}{2} \alpha_{1} b^{2}-\frac{1}{2} \varepsilon a^{2}\right) y^{2}+\left[-\frac{1}{6} \beta_{1} \varepsilon b a^{2}\right.
$$




$$
\begin{aligned}
& \left.-\alpha_{1} b\left(-\frac{1}{2} \alpha_{1} b^{2}-\frac{1}{2} \varepsilon a^{2}\right)-\frac{1}{3} \alpha_{2} b^{3}\right] y^{3} \\
& -\left\{\frac{1}{2} \varepsilon a\left[\frac{1}{3} \beta_{1}^{2} a b^{2}+\frac{1}{3} \beta_{1}\left(-\frac{1}{2} \alpha_{1} b^{2}-\frac{1}{2} \varepsilon a^{2}\right) a\right]\right. \\
& -\alpha_{1} b\left[-\frac{1}{6} \beta_{1} \varepsilon b a^{2}-\alpha_{1} b\left(-\frac{1}{2} \alpha_{1} b^{2}-\frac{1}{2} \varepsilon a^{2}\right)\right. \\
& -\frac{1}{3} \alpha_{2} b^{3}-\frac{1}{2} \alpha_{1}\left(-\frac{1}{2} \alpha_{1} b^{2}-\frac{1}{2} \varepsilon a^{2}\right) \\
& -\alpha_{2}\left(-\frac{1}{2} \alpha_{1} b^{2}-\frac{1}{2} \varepsilon a^{2}\right) b^{2}+\frac{1}{12} \varepsilon \beta_{1}^{2} b^{2} a^{2} \\
& \left.+\frac{1}{12} \beta_{1} \varepsilon\left(-\frac{1}{2} \alpha_{1} b^{2}-\frac{1}{2} \varepsilon a^{2}\right) a^{2}\right]+\ldots
\end{aligned}
$$

and

$$
\begin{aligned}
& U(y)=a y+\frac{1}{2} \beta_{1} b a y^{2}+\left[\frac{1}{3} \beta_{1}^{2} a b^{2}+\frac{1}{3} \beta_{1}\left(-\frac{1}{2} \alpha_{1} b^{2}\right.\right. \\
& \left.\left.-\frac{1}{2} \varepsilon a^{2}\right) a\right] y^{3}+\left\{\frac { 3 } { 4 } \beta _ { 1 } b \left[\frac{1}{3} \beta_{1}^{2} a b^{2}+\frac{1}{3} \beta_{1}\left(-\frac{1}{2} \alpha_{1} b^{2}\right.\right.\right. \\
& \left.-\frac{1}{2} \varepsilon a^{2}\right) a+\frac{1}{4} \beta_{1}^{2}\left(-\frac{1}{2} \alpha_{1} b^{2}-\frac{1}{2} \varepsilon a^{2}\right) b a \\
& +\frac{1}{4} \beta_{1}\left\{\left[-\frac{1}{6} \beta_{1} \varepsilon b a^{2}-\alpha_{1} b\left(-\frac{1}{2} \alpha_{1} b^{2}-\frac{1}{2} \varepsilon a^{2}\right)\right.\right. \\
& \left.\left.-\frac{1}{3} \alpha_{2} b^{3}\right] a\right\} y^{4}+\ldots
\end{aligned}
$$

Substitution of boundary condition (28) into Eq. (33) gives us $a$ and $b$ through the following equations:

$$
\begin{aligned}
& \theta(1)=b-\frac{1}{2} \alpha_{1} b^{2}-\frac{1}{2} \varepsilon a^{2}-\frac{1}{6} \beta_{1} \varepsilon b a^{2}+\frac{1}{2} \alpha_{1}^{2} b^{3} \\
& +\frac{1}{2} \alpha_{1} \varepsilon b a^{2}-\frac{1}{3} \alpha_{2} b^{3}-\frac{1}{12} \varepsilon \beta_{1}^{2} b^{2} a^{2} \\
& +\frac{5}{24} \varepsilon a^{2} \beta_{1} \alpha_{1} b^{2}+\frac{1}{24} \varepsilon^{2} a^{4} \beta_{1}-\frac{5}{8} \alpha_{1}^{3} b^{4}-\frac{3}{4} \alpha_{1}^{2} b^{2} \varepsilon a^{2} \\
& +\frac{5}{6} \alpha_{1} \alpha_{2} b^{4}-\frac{1}{8} \alpha_{1} \varepsilon^{2} a^{4}+\frac{1}{2} \alpha_{2} b^{2} \varepsilon a^{2}+\ldots=0
\end{aligned}
$$

and

$$
\begin{gathered}
U(1)=a+\frac{1}{2} \beta_{1} b a+\frac{1}{3} \beta_{1}^{2} b^{2} a-\frac{1}{6} \beta_{1} a \alpha_{1} b^{2}-\frac{1}{6} \beta_{1} a^{3} \varepsilon \\
+\frac{1}{4} \beta_{1}^{3} b^{3} a-\frac{1}{4} \beta_{1}^{2} b^{3} a \alpha_{1}-\frac{7}{24} \beta_{1}^{2} \varepsilon a^{3} b+\frac{1}{8} \beta_{1} a \alpha_{1}^{2} b^{3} \\
+\frac{1}{8} \beta_{1} a^{3} \alpha_{1} b \varepsilon-\frac{1}{12} \beta_{1} a \alpha_{2} b^{3}+\ldots=1 .
\end{gathered}
$$

Solving of Eqs. (35) and (36) gives us the value of $a$ and $b$.
These values are too long to be shown in this paper. By substituting $a$ and $b$ obtained above into Eq. (33) and Eq. (34) we can find the expressions of $\theta(y)$ and $U(y)$.

\section{Results and discussions}

The comparison of the results obtained by four methods for different values of the perturbation parameters in the range $0.2-0.6$ are shown in Figs. $2-5$.

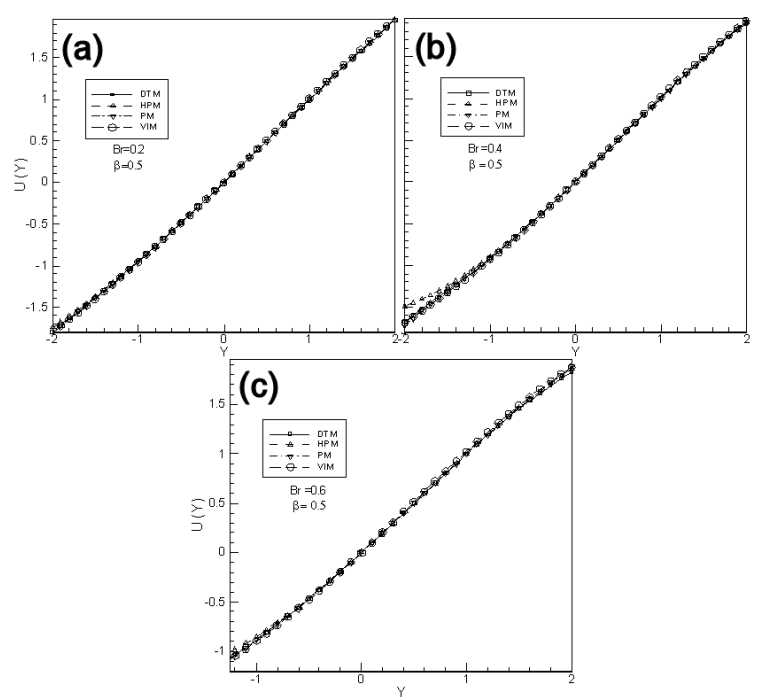

Fig. 2. A comparison of the $U(Y)$ of DTM and three other methods for Example 1, at $B r=0.2$ (a), 0.4 (b), $0.6(\mathrm{c})$.

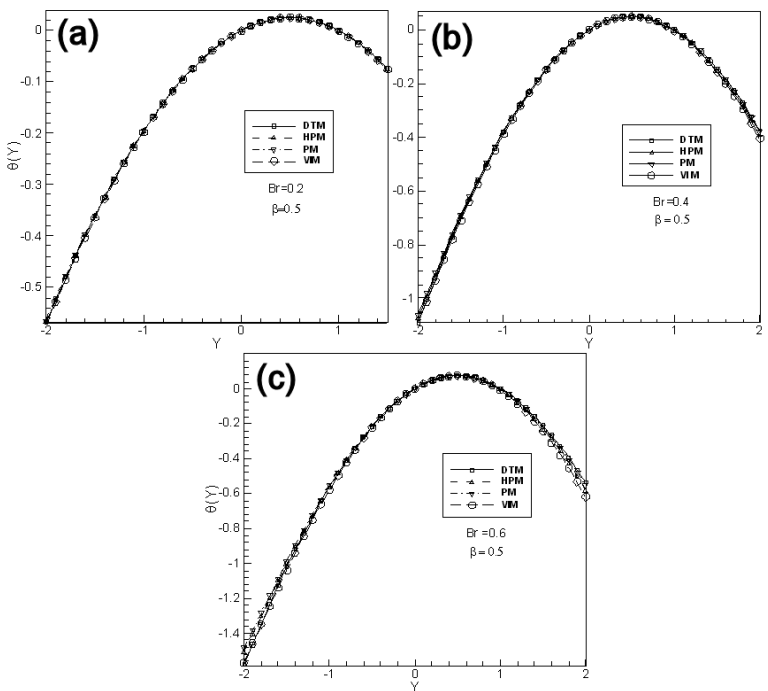

Fig. 3. A comparison of the $\theta(Y)$ of DTM and three other methods for Example 1, at $B r=0.2$ (a), 0.4 (b), $0.6(\mathrm{c})$.

In a special case in which perturbation parameter is equal to 0.4 , results of the present analysis are tabulated 


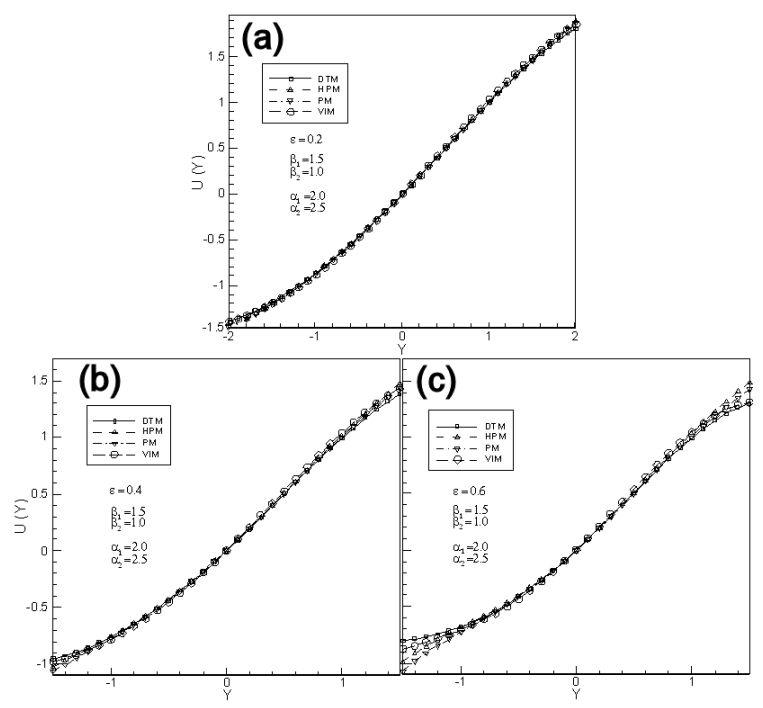

Fig. 4. A comparison of the $U(Y)$ of DTM and three other methods for Example 2, at $\varepsilon=0.2$ (a), 0.4 (b), $0.6(\mathrm{c})$.

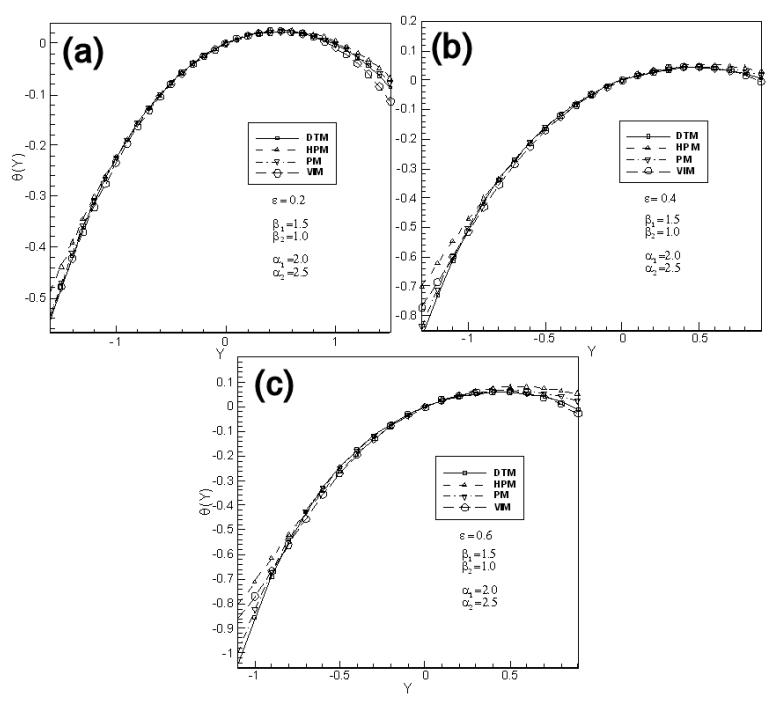

Fig. 5. A comparison of the $\theta(Y)$ of DTM and three other methods for Example 2, at $\varepsilon=0.2$ (a), 0.4 (b), $0.6(\mathrm{c})$.

against the numerical solution in Tables II-V. A very interesting agreement between the results is observed, which confirms the validity of the DTM.

\section{Conclusions}

In this paper the differential transformation method is applied to solve the systems of nonlinear equations arising in fluid flows with variable viscosity. Throughout the results of two examples which are found by using the DTM are compared with three other methods such as HP, VI, and $\mathrm{P}$, we showed that the convergence is quite close.
TABLE II

The results of DTM and numerical solution for $U(Y)$ for Example 1.

\begin{tabular}{c|c|c|c}
\hline \hline \multicolumn{4}{|c}{$B r=0.4, \beta=0.5$} \\
\hline$Y$ & Numerical & DTM & Error \\
\hline 0 & 0 & 0 & 0 \\
0.1 & 0.098804 & 0.098982594 & 0.001803 \\
0.2 & 0.198398 & 0.198760212 & 0.001825 \\
0.3 & 0.298593 & 0.299144039 & 0.001844 \\
0.4 & 0.399195 & 0.399936115 & 0.001858 \\
0.5 & 0.5 & 0.500931104 & 0.001862 \\
0.6 & 0.600805 & 0.601918053 & 0.001852 \\
0.7 & 0.701407 & 0.702682154 & 0.001819 \\
0.8 & 0.801602 & 0.803006505 & 0.001752 \\
0.9 & 0.901196 & 0.902673877 & 0.00164 \\
1 & 1 & 1.001468468 & 0.001468
\end{tabular}

TABLE III

The results of DTM and numerical solution for $\theta(Y)$ for Example 1.

\begin{tabular}{c|c|c|c}
\hline \hline \multicolumn{4}{c}{$B r=0.4, \beta=0.5$} \\
\hline$Y$ & Numerical & DTM & Error \\
\hline 0 & 0 & 0 & 0 \\
0.1 & 0.017729362 & 0.017824005 & 0.005338215 \\
0.2 & 0.031555656 & 0.031730851 & 0.00555194 \\
0.3 & 0.041451472 & 0.041692946 & 0.005825467 \\
0.4 & 0.047396975 & 0.047690477 & 0.006192426 \\
0.5 & 0.049380152 & 0.049711905 & 0.00671835 \\
0.6 & 0.047396975 & 0.047754468 & 0.007542541 \\
0.7 & 0.041451472 & 0.041824681 & 0.009003504 \\
0.8 & 0.031555656 & 0.03193883 & 0.012142798 \\
0.9 & 0.017729362 & 0.018123476 & 0.022229479
\end{tabular}

TABLE IV

The results of DTM and numerical solution for $U(Y)$ for Example 2.

\begin{tabular}{c|c|c|c}
\hline \hline \multicolumn{4}{c}{$\varepsilon=0.4, \beta_{1}=1.5, \beta_{2}=1, \alpha_{1}=2, \alpha_{2}=2.5$} \\
\hline$Y$ & Numerical & DTM & Error \\
\hline 0 & 0 & 0 & 0 \\
0.1 & 0.096605 & 0.097138 & 0.005513 \\
0.2 & 0.195446 & 0.196462 & 0.0052 \\
0.3 & 0.295997 & 0.29743 & 0.004842 \\
0.4 & 0.397706 & 0.399459 & 0.004407 \\
0.5 & 0.5 & 0.501923 & 0.003845 \\
0.6 & 0.602294 & 0.604152 & 0.003086 \\
0.7 & 0.704003 & 0.705438 & 0.002039 \\
0.8 & 0.804554 & 0.805028 & 0.000588 \\
0.9 & 0.903395 & 0.902126 & 0.0014 \\
1 & 1 & 0.995897 & 0.0041
\end{tabular}


TABLE V

The results of DTM and numerical solution for $\theta(Y)$ for Example 2.

\begin{tabular}{c|c|c|c}
\hline \hline \multicolumn{3}{c}{$\varepsilon=0.4, \beta_{1}=1.5, \beta_{2}=1, \alpha_{1}=2, \alpha_{2}=2.5$} \\
\hline$Y$ & Numerical & DTM & Error \\
\hline 0 & 0 & 0 & 0 \\
0.1 & 0.01694 & 0.016566 & 0.022083 \\
0.2 & 0.029822 & 0.02905 & 0.025903 \\
0.3 & 0.038873 & 0.037652 & 0.031428 \\
0.4 & 0.044246 & 0.042467 & 0.040191 \\
0.5 & 0.046027 & 0.043489 & 0.055144 \\
0.6 & 0.044246 & 0.040604 & 0.082304 \\
0.7 & 0.038873 & 0.033598 & 0.135718 \\
0.8 & 0.029822 & 0.02215 & 0.257268 \\
0.9 & 0.01694 & 0.005838 & 0.655382
\end{tabular}

Therefore, this method can be applied to many complicated linear and nonlinear partial differential equations and system of partial differential equations and does not require linearization, discretization, or perturbation.

\section{References}

[1] W.E. Boyce, Elementary Differential Equations and Boundary Value Problems, Wiley, New York 1977.

[2] L. Debnath, Nonlinear Partial Differential Equations for Scientists and Engineers, Burghausen, Berlin 1998.

[3] R. Dennemeyer, Introduction to Partial Differential Equations and Boundary Value Problems, California State College at San Bernardino, New York 1968.

[4] W.A. Strauss, Partial Differential Equations. An Introduction, Wiley, Singapore 1992.

[5] E. Zauderer, Partial Differential Equations of Applied Mathematics, Wiley, USA 1983.

[6] J.K. Zhou, Differential Transformation and Its Applications for Electrical Circuits, Huarjung University Press, Wuuhahn, China 1986 (in Chinese).

[7] J.S. Chiou, J.R. Tzeng, Trans. ASME J. Vib. Acoust. 118, 83 (1996).
[8] M.J. Jang, C.L. Chen, Y.C. Liu, Appl. Math. Comput. 121, 261 (2001).

[9] I.H. Abdel-Halim Hassan, Appl. Math. Comput. 127, 1 (2002).

[10] M. Omidvar, A. Barari, M. Momeni, D.D. Ganji, Geomech. Geoeng. Int. J. 5, 127 (2010).

[11] S.S. Ganji, A. Barari, L.B. Ibsen, G. Domairry, Central Europ. J. Operat. Res. 20, 87 (2012).

[12] A.A. Joneidi, D.D. Ganji, M. Babaelahi, Int. Commun. Heat Mass Transfer 36, 757 (2009).

[13] Y.L. Yeh, C.C. Wang, M.J. Jang, Appl. Math. Comput. 190, 1146 (2007).

[14] C. Wang, Y.H.J. Au, Mech. Sci. 2, 147 (2011).

[15] F. Fouladi, E. Hosseinzadeh, A. Barari, G. Domairry, J. Heat Transfer Res. 41, 155 (2010).

[16] M.O. Miansari, M.E. Miansari, A. Barari, G. Domairry, Int. J. Comput. Methods Eng. Sci. Mech. 11, 79 (2010).

[17] H. Mirgolbabaei, A. Barari, L.B. Ibsen, M.G. Sfahani, Arch. Civil Mech. Eng. 10, 41 (2010).

[18] M. Rahimi, M.J. Hosseini, A. Barari, G. Domairry, M. Ebrahimpour, Tehnicki Vjesnik 18, 315 (2011).

[19] A. Aziz, S.M. Enamul Hug, ASME J. Heat Transfer 97, 300 (1975).

[20] A.R. Ghotbi, M. Omidvar, A. Barari, Comput. Geotechn. 38, 777 (2011).

[21] M. Momeni, N. Jamshidi, A. Barari, G. Domairry, Int. J. Numeric Meth. Heat Fluid. Flow 21, 206 (2011).

[22] H. Bararnia, E. Ghasemi, S. Soleimani, A. Barari, D.D. Ganji, J. Porous Media 14, 545 (2011).

[23] A.G. Dunning, N. Tolou, J.L. Herder, Mech. Sci. 2, 157 (2011).

[24] E. Hosseinzadeh, A. Barari, F. Fouladi, G. Domairry, Therm. Sci. 14, 1101 (2010).

[25] D.D. Gganji, S.M. Varedi, M. Rahimi, J. Heat Mass- Transfer 30, 287 (2008).

[26] R. Keslerová, K. Kozel, Math. Comput. Simulation 80, 1783 (2010).

[27] A. Aziz, T.Y. Na, Perturbation Method in Heat Transfer, Hemisphere Publishing Corporation, Washington, DC 1984. 